\title{
TOXICIDAD ORAL A 60 DÍAS DEL ACEITE DE SACHA INCHI (Plukenetia volubilis L.) Y LINAZA (Linum usitatissimum L.) Y DETERMINACIÓN DE LA DOSIS LETAL 50 EN ROEDORES
}

\author{
Arilmi Gorriti ${ }^{1,2, a, b}$, Jorge Arroyo ${ }^{3, a}$, Fredy Quispe ${ }^{4, a, c}$, Braulio Cisneros ${ }^{3, a}$, \\ Martín Condorhuamán ${ }^{5, d}$, Yuan Almora ${ }^{6, a}$, Víctor Chumpitaz
}

\begin{abstract}
RESUMEN
Objetivos. Evaluar la toxicidad oral a 60 días y determinar la dosis letal 50 (DL 50) de los aceites crudos de sacha inchi (Plukenetia volubilis L.) y linaza (Linum ussitatisimum) en ratas Holtzman y en ratones cepa Balb C57, respectivamente. Materiales y métodos. Para la evaluación de la toxicidad oral a dosis repetida por 60 días se utilizó 24 ratas macho Holtzman divididos en tres grupos de ocho cada uno, los grupos fueron: solución salina fisiológica $4 \mathrm{~mL} / \mathrm{kg}$ (SSF), aceite de sacha inchi $0,5 \mathrm{~mL} / \mathrm{kg}$ (SI05) y aceite de linaza $0,5 \mathrm{~mL} / \mathrm{kg}$ (L05), durante el experimento se controló semanalmente el peso corporal y signos de toxicidad en los grupos investigados, así como colesterol total, HDL, triglicéridos, glucosa, urea, TGP y fosfatasa alcalina a los 30 y 60 días de iniciado el experimento. Para la evaluación de la DL50 se usó ratones macho cepa Balb C57 en grupos de diez animales, se administró por vía oral dosis crecientes de aceites crudos hasta alcanzar $1 \mathrm{~mL} / \mathrm{kg}$ (37 g/kg); Resultados. Los parámetros séricos en las ratas indican que no existe toxicidad alguna a los 60 días y que la administración de los aceites disminuyeron los niveles de colesterol, triglicéridos e incrementaron el HDL con respecto al grupo control. La DL50 muestra que los aceites crudos de sacha inchi y linaza presentan dosis por encima de los $37 \mathrm{~g} / \mathrm{kg}$ de masa corporal. Conclusiones. Los aceites de sacha inchi y linaza son inocuos a 60 días y presentan una DL50 por encima de los $37 \mathrm{~g} / \mathrm{kg}$ de animal.
\end{abstract}

Palabras clave: Farmacología, Plantas medicinales, Pruebas de toxicidad, Dosificación letal mediana (fuente: DeCS BIREME).

\section{ORAL TOXICITY AT 60-DAYS OF SACHA INCHI OIL (Plukenetia volubilis L.) AND LINSEED (Linum usitatissimum L.), AND DETERMINATION OF LETHAL DOSE 50 IN RODENTS}

\begin{abstract}
Objectives. To evaluate the oral toxicity at 60 days and to determine the lethal dose 50 (LD 50) of raw sacha inchi (Plukenetia volubilis L.) and linseed (Linum ussitatisimum) oils in Holtzman rats and mice of the strain Balb C57 respectively. Materials and methods. For the evaluation of the oral toxicity of repeated doses for 60 days, 24 male Holtzman rats were used, divided in three groups of 8 each, the groups were: physiologic saline solution $4 \mathrm{~mL} / \mathrm{kg}$ (FSS), sacha inchi oil $0.5 \mathrm{~mL} / \mathrm{kg}$ (SI05) and linseed oil $0.5 \mathrm{~mL} / \mathrm{kg}$ (L05), during the experiment the body weight was controlled weekly, and signs of toxicity in the research groups, as well as total cholesterol, HDL, glucose, triglycerides and alkaline phosphatase at days 30 and 60 after initiating the experiment. For the evaluation of the LD50 male mice of the Balb C57 strain were used in groups of 10 animals, and they were administered increasing oral doses of raw oils until reaching $1 \mathrm{~mL} / \mathrm{kg}(37 \mathrm{~g} / \mathrm{kg})$. Results. The serum parameters in the rats indicated there is no toxicity at 60 days and that the administration of the oils lowered the levels of cholesterol, triglycerides and increased the HDL in comparison with the control group. The LD50 shows that the raw sacha inchi and linseed oils have doses above $37 \mathrm{~g} / \mathrm{kg}$ of body weight. Conclusions. Sacha inchi and linseed oils are harmless at 60 days and present a LD50 above the $37 \mathrm{~g} / \mathrm{kg}$ of animal.
\end{abstract}

Key words: Pharmacology; Plants, medicinal; Toxicity tests; Lethal dose 50 (source: MeSH NLM).

\footnotetext{
Laboratorio de Farmacognosia y Medicina Tradicional, Facultad de Farmacia y Bioquímica, Universidad Nacional Mayor de San Marcos. Lima, Perú.

2 Centro Latinoamericano de Enseñanza e Investigación Bacteriológica, Facultad de Farmacia y Bioquímica, Universidad Nacional Mayor de San Marcos. Lima, Perú.

Instituto de Investigaciones Clínicas, Facultad de Medicina, Universidad Nacional Mayor de San Marcos. Lima, Perú

Unidad de I-D+I, Empresa Agronegocios Peruagro S.R.L. Arequipa, Perú.

Dirección de Salud (DISA) V, Ministerio de Salud. Lima, Perú.

Departamento de Farmacia, Hospital Nacional Hipólito Unánue. Lima, Perú.

Laboratorio de Farmacología, Facultad de Odontología, Universidad Nacional Mayor de San Marcos. Lima, Perú.

Químico Farmacéutico; ' Doctor en Farmacia y Bioquímica; ' ${ }^{\circ}$ Candidato a Magíster en Química; d Médico Cirujano.
}

Recibido: 19-02-10 Aprobado: 01-09-10 


\section{INTRODUCCIÓN}

Elsachainchi(PlukenetiavolubilisL.)esunaEuphorbiaceae distribuida desde América Central hasta Bolivia, en el caso de Perú, se encuentra fundamentalmente en la selva alta y baja, en alturas que van desde los 100 hasta los 2000 metros de altura, en zonas con precipitaciones anuales promedio de $1084 \mathrm{~mm}^{3}$ y temperaturas que oscilan entre los 10 y $36,6{ }^{\circ} \mathrm{C}{ }^{(1-3)}$. Investigaciones realizadas en esta planta revelan contenidos superiores de aceite con respecto a las semillas de soya, maíz, maní, girasol, algodón, palma y oliva ${ }^{(4)}$; el análisis de ácidos grasos del aceite revelan contenidos de ácido linoléico comparables al aceite de maní, ácido a-linolénico (AAL) superiores a los aceites de soya, maíz, maní, girasol, algodón, palma y oliva ${ }^{(4)}$, y contenidos inferiores en AAL y ácido oleico al presentado por el aceite de linaza ${ }^{(3)}$.

Tanto el sacha inchi como la linaza presentan cantidades considerables de ácidos grasos poliinsaturados (PUFA), los cuales son esenciales para el organismo humano $(5,6)$. Al respecto, se ha descrito numerosos efectos cardioprotectores, antiarrítimicos, antiinflamatorios, hipotensores, neuroprotectores, hipotriglicerodémicos e hipocolesterolémicos por parte del AAL, así como su rol esencial en el organismo como precursor de ácidos grasos de cadena más larga EPA (ácido eicosapentaenoico) y DHA (ácido docosahexaenoico). Entre las fuentes ricas de AAL se encuentran la linaza, canola y colza, siendo la primera, la fuente comercial más importante a nivel mundial con numerosos estudios ${ }^{(6,7)}$.

En Perú, el sacha inchi se viene comercializando y consumiendo en base a los potenciales efectos benéficos que puede tener, sin embargo, no se cuenta con estudios que evalúen su toxicidad. En ese sentido, la presente investigación tiene como objetivos evaluar comparativamente la toxicidad oral a 60 días del aceite de sacha inchi y linaza, asimismo, determinar la DL50 de los aceites crudos.

\section{MATERIALES Y MÉTODOS}

\section{TIPO DE ESTUDIO}

Se realizó un estudio experimental dividido en dos grandes secciones: la evaluación de toxicidad y la determinación de dosis letal media en modelos animales con ratas Holtzman y ratones cepa Balb C57, respectivamente.

\section{RECOLECCIÓN DE LAS SEMILLAS}

El aceite crudo de sacha inchi se obtuvo a partir de semillas recolectadas en la región San Martín, en la zona noroeste de Perú, a aproximadamente 379 metros de altura, entre los meses de febrero y marzo de 2009. El aceite crudo de linaza se obtuvo a partir de semillas adquiridas en el mercado Central "La Parada" de Lima, Perú. Ambas muestras fueron identificadas taxonómicamente.

\section{OBTENCIÓN DE LOS ACEITES CRUDOS DE SACHA INCHI Y LINAZA}

Alrededor de $200 \mathrm{~g}$ de almendras o semillas convenientemente seleccionadas se colocaron en el cilindro de acero inoxidable de una prensa hidráulica (marca nacional) luego se procedió a prensar hasta alcanzar la presión de 3000 psi. Se decantó los aceites crudos obtenidos y, posteriormente, se filtró a través de papel filtro (Whatman $\mathrm{N}^{\circ}{ }^{\circ}$ ) con la ayuda de una bomba de vacío (Copelametic, USA), los volúmenes filtrados se almacenaron a 5 ${ }^{\circ} \mathrm{C}$ en frascos de color ámbar. Es preciso mencionar que todos los reactivos y solventes utilizados fueron de grado analítico Sigma Aldrich Chemical Co. y Merck.

\section{ANÁLISIS DE LOS ACEITES CRUDOS}

Los aceites crudos se evaluaron según los métodos: índice de acidez método AOAC ${ }^{(8)}(1990,940.28)$, índice de yodo método $\operatorname{AOAC}^{(8)}(1990,920.159)$, índice de peróxido método AOAC ${ }^{(8)}(1990,965.33)$, densidad en el densitómetro (Mettler Toledo Densito 30P, USA) e índice de refracción en el refractómetro (Mettler Toledo Modelo 30PX, USA). Los ácidos grasos del aceite de sacha inchi fueron convertidos a su correspondientes ésteres de metilo y evaluados por cromatografía de gases de acuerdo con el método validado por el Instituto Tecnológico Pesquero del Perú (LABS-ITP-FQ-002-98, 2003) ${ }^{(9)}$.

\section{EVALUACIÓN DE LA TOXICIDAD ORAL REPETIDA A LOS 60 DÍAS EN RATAS HOLTZMAN}

Se utilizó 24 ratas albinas macho de la cepa Holtzman con pesos promedio de $100 \pm 20$ gramos procedentes del Instituto Nacional de Salud de Perú (INS), los que se mantuvieron en el Bioterio de la Facultad de Medicina de la Universidad Nacional Mayor de San Marcos, el experimento se inició con un condicionamiento previo de 48 horas, con agua y alimento ad libitum, ciclo luz-día de 12 horas y temperatura entre 22 y $26^{\circ} \mathrm{C}$.

El modelo experimental contempló un seguimiento por 60 días a tres grupos de ocho ratas cada uno: grupo control normal que recibió solución salina fisiológica $4 \mathrm{~mL} / \mathrm{kg}$ (SSF), grupo aceite de sacha inchi $0,5 \mathrm{~mL}$ (SI05) y grupo aceite de linaza $0,5 \mathrm{~mL}$ (L05). La evaluación de la toxicidad oral repetida se realizó a través de evaluaciones del peso corporal $(\mathrm{g})$ y sus resultados 
permitieron determinar el incremento del peso corporal a través de la siguiente expresión:

$\operatorname{IPC}(\%)=\left(\left(P_{T}-P_{0}\right) / P_{0}\right) * 100$

Donde $I P C=$ representa el incremento del peso corporal $(I P C)$ en $\%, P_{T}=$ peso del grupo en estudio en un tiempo $\mathrm{T} ; P_{0}=$ Peso del grupo en estudio en tiempo inicial ${ }^{(11-12)}$. Para determinar el efecto acumulativo tóxico de los aceites crudos de sacha inchi y linaza sobre los animales, se realizó evaluaciones bioquímicas de colesterol total, HDL, triglicéridos, urea, glucosa, TGP y fosfatasa alcalina según métodos enzimáticos de acuerdo con recomendaciones internacionales ${ }^{(13)}$ a los 30 y 60 días del experimento.

\section{EVALUACIÓN DE LA DL50 EN RATONES ${ }^{(10)}$}

Se utilizó 210 ratones macho de la cepa Balb C57 con peso promedio de $20 \pm 3 \mathrm{~g}$ procedentes del Instituto Nacional de Salud (Lima, Perú), los cuales fueron distribuidos aleatoriamente en 21 grupos de diez animales cada uno, según el método de Vega y Carrillo ${ }^{(10)}$ y de acuerdo con las recomendaciones para el cuidado y empleo de animales en el laboratorio (Guide for the Care and Use of Laboratory Animals) ${ }^{(11,12)}$. Después de una semana de aclimatación y alimentación con ratonina peletizada y agua a voluntad, previo ayuno de cuatro horas, se les administró por vía oral: solución de suero fisiológico 10 $\mathrm{mL} / \mathrm{kg}$ a un grupo, y a los 20 grupos restantes aceite de sacha inchi y linaza en cantidades crecientes de 0,1 ; 0,$2 ; 0,3 ; 0,4 ; 0,5 ; 0,6 ; 0,7 ; 0,8 ; 0,9$ y $1 \mathrm{~mL}$ por ratón. Los animales fueron observados constantemente durante las primeras 24 horas y luego diariamente durante un periodo de 14 días registrándose cualquier síntoma tóxico que pudiera presentarse, con el número de muertes en cada grupo se calculó la DL50 empleando el método Probit.

\section{ANÁLISIS DE DATOS}

Los datos de los pesos corporales obtenidos semanalmente y de los parámetros séricos de colesterol total, HDL, triglicéridos, glucosa, úrea, TGP y fosfatasa alcalina a los 30 y 60 días se evaluaron en el software estadístico SAS ${ }^{\circledR}$ versión 7 (SAS Institute Inc.). Las variables numéricas se describieron con medidas de tendencia central y de dispersión, media y desviación estándar, respectivamente.

Se evaluó la normalidad y homogeneidad de varianzas, por medio de las pruebas de Shapiro Wilk y Barlet; respectivamente. Posteriormente se efectuó un análisis de varianza (ANOVA), con pruebas post hoc de Tukey en aquellas variables donde la diferencia entre los grupos fue significativa $p<0,05$.
Tabla 1. Características fisicoquímicas de los aceites crudos de sacha inchi y linaza.

\begin{tabular}{lcc}
\hline & \multicolumn{2}{c}{ Aceite crudo } \\
\cline { 2 - 3 } $\begin{array}{l}\text { Característica } \\
\text { fisicoquímica }\end{array}$ & Sacha inchi* & Linaza * $^{*}$ \\
\hline Índice de refracción & $1,480 \pm 0,000$ & $1,478^{(14)}$ \\
\hline $\begin{array}{l}\text { Densidad a } 25^{\circ} \mathrm{C} \\
\text { (g/mL) }\end{array}$ & $0,9269 \pm 0,002$ & $0,931^{(14)}$ \\
$\begin{array}{l}\text { Ácidos grasos libres } \\
\text { (\% ácido oleico) }\end{array}$ & $0,231 \pm 0,003$ & $0,304 \pm 0,028$ \\
$\begin{array}{l}\text { Índice de yodo } \\
\text { (g de I/ 100g) }\end{array}$ & $194,035 \pm 0,381$ & $188,707 \pm 0,189$ \\
$\begin{array}{l}\text { Índice de saponificación } \\
\text { (mg KOH/g) }\end{array}$ & $184,748 \pm 0,200$ & $197,976 \pm 0,480$ \\
\hline $\begin{array}{l}\text { Valor de peróxido } \\
\text { (meq O/ kg) }\end{array}$ & $0,889 \pm 0,143$ & $0,396 \pm 0,280$ \\
\hline $\begin{array}{l}\text { Valor de dienos } \\
\text { conjugados (umol/g) }\end{array}$ & $7,282 \pm 0,524$ & $8,459 \pm 1,457$ \\
\hline
\end{tabular}

Media de dos repeticiones \pm desviación estándar.

\section{RESULTADOS}

\section{CARACTERÍSTICAS QUIMMICAS DE LOS ACEITES CRUDOS}

Las características químicas de los aceites crudos de sacha inchi y linaza se describen en la Tabla 1, donde se presenta los valores de índice de refracción, densidad, ácidos grasos libres, índice de yodo, índice de saponificación, valor de peróxido y dienos conjugados de los aceites crudos. Para el caso del índice de refracción y densidad del aceite de linaza se consideró el valor reportado por Biswas et al. ${ }^{(14)}$.

El contenido de ácidos grasos saturados e insaturados del aceite crudo de sacha inchi después de ser decantado, filtrado y deshidratado se analizó en el cromatógrafo de gases, previa conversión a ésteres de metilo (Tabla 2) ${ }^{(15-18)}$.

\section{TOXICIDAD ORAL A 60 DÍAS DE LOS ACEITES CRUDOS}

La evaluación de la toxicidad oral de los aceites crudos de sacha inchi y linaza a 60 días, se realizó a partir de la observación directa de los animales en los grupos estudiados, acompañada del registro de sus pesos corporales.

En la Tabla 3 se presenta la evaluación del peso corporal de los animales en los diferentes grupos hacia las ocho semanas, la tabla muestra que los grupos constituidos por la solución salina de suero fisiológico (SSF), así 
Tabla 2. Contenido de ácidos grasos del aceite de sacha inchi (SI) y de linaza (L) comparado con otros estudios ${ }^{(15-18)}$.

\begin{tabular}{|c|c|c|c|c|c|}
\hline \multirow[b]{2}{*}{ Ácidos grasos (\%) } & \multicolumn{3}{|c|}{ Sacha Inchi } & \multicolumn{2}{|c|}{ Linaza } \\
\hline & Gorriti & Hamaker (15) & Follegatti ${ }^{(16)}$ & Rallidis $^{(17)}$ & Clandinin $^{(18)}$ \\
\hline Ácido mirístico (C14:0) & - & - & - & - & 0,2 \\
\hline Ácido palmítico (C16:0) & $3,95 \pm 0,01$ & 4,50 & 4,24 & 5,9 & 8,6 \\
\hline Ácido esteárico (C18:0) & $2,97 \pm 0,01$ & 3,20 & 2,50 & 3,6 & 3,3 \\
\hline Ácido oleico (C18:1) & $9,01 \pm 0,01$ & 9,60 & 8,41 & 18,2 & 15,8 \\
\hline Ácido vacénico & $0,58 \pm 0,01$ & - & - & - & - \\
\hline Ácido linoléico (C18:2) & $36,19 \pm 0,02$ & 36,80 & 34,08 & 13,9 & 14,2 \\
\hline Ácido a-linolénico (C18:3) & $47,06 \pm 0,02$ & 45,20 & 50,41 & 54,2 & 57,5 \\
\hline Ácido eicosaenoico & $0,27 \pm 0,03$ & - & - & - & - \\
\hline Ácidos saturados & $6,91 \pm 0,01$ & 7,70 & 6,74 & 9,5 & 11,9 \\
\hline Ácido monoinsaturados & $9,86 \pm 0,02$ & 9,60 & 8,41 & 18,2 & 15,8 \\
\hline Ácido poliinsaturados & $83,24 \pm 0,01$ & 82,00 & 84,49 & 68,1 & 71,7 \\
\hline Relación omega-3/omega-6 & 1,30 & 1,25 & 1,48 & 3,89 & 4,05 \\
\hline
\end{tabular}

Media de dos repeticiones \pm desviación estándar.

como los constituidos por aceite de sacha inchi en la dosis de $0,5 \mathrm{~mL}$ (SI05) y aceite de linaza en la dosis de $0,5 \mathrm{~mL}$ (L05) no presentaron diferencias significativas del peso corporal entre ellos, durante las ocho semanas del experimento. Asimismo, se presentan los cambios a través del tiempo para los tres grupos en la Figura 1.

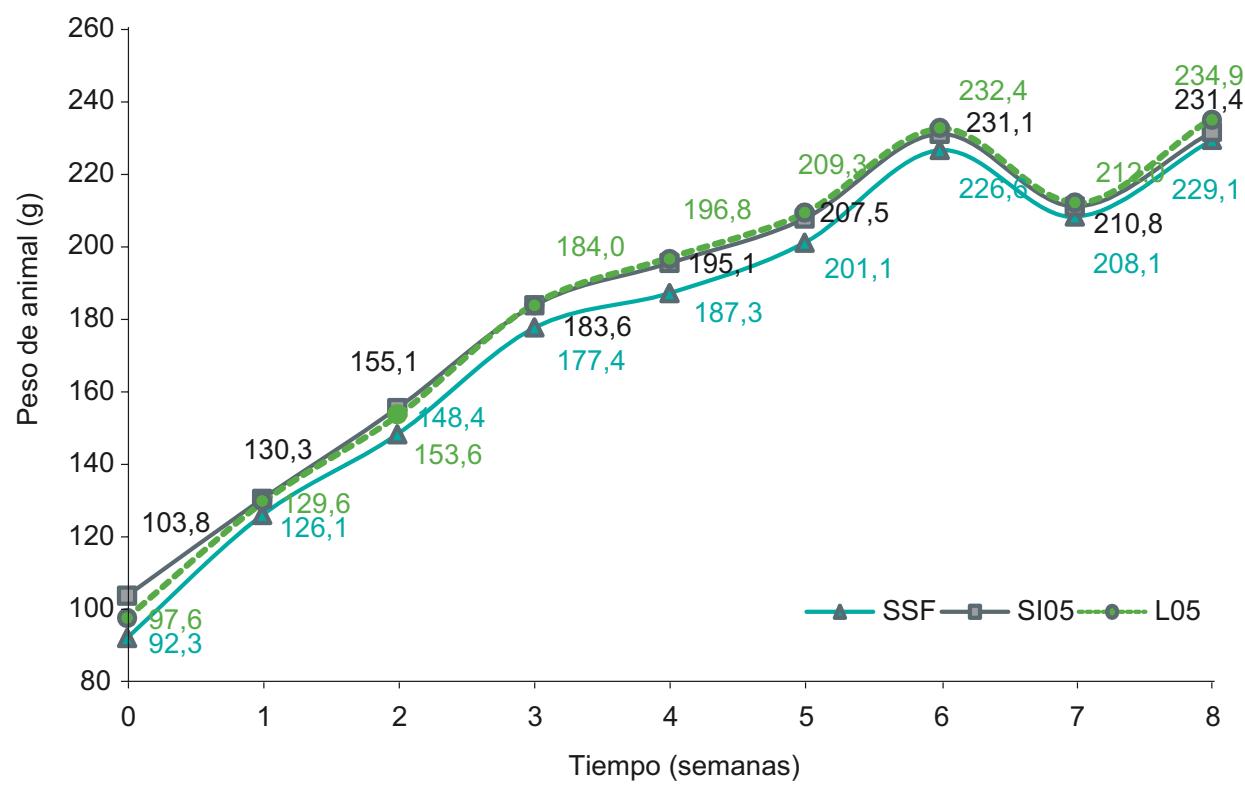

Figura 1. Peso corporal de los animales en los diferentes grupos. SSF: Solución salina de suero fisiológico, SI05: aceite de sacha inchi 0,5 mL, L05: aceite de linaza 0,5 mL. Cada punto representa valores promedio de ocho animales.

Tabla 3. Peso corporal de los animales en los diferentes grupos durante 60 días.

\begin{tabular}{|c|c|c|c|c|c|c|c|c|c|}
\hline \multirow{2}{*}{ Grupos } & \multicolumn{9}{|c|}{ Peso de animal - semana (g) } \\
\hline & $0^{\text {ns }}$ & 1 & 2 & 3 & 4 & 5 & 6 & 7 & 8 \\
\hline $\begin{array}{l}\text { Suero } \\
\text { fisiológico } \\
(n=8)^{*}\end{array}$ & $92,3 \pm 9,8$ & $126,1 \pm 7,5$ & $148,4 \pm 8,8$ & $177,4 \pm 10,7$ & $187,3 \pm 13,6$ & $201,1 \pm 12,3$ & $226,6 \pm 13,9$ & $208,1 \pm 18,7$ & $229,1 \pm 23,2$ \\
\hline $\begin{array}{l}\text { Aceite sacha } \\
\text { inchi }(n=8)^{*}\end{array}$ & $103,8 \pm 11,5$ & $130,3 \pm 15,6$ & $155,1 \pm 14,8$ & $183,6 \pm 8,4$ & $195,1 \pm 17,9$ & $207,5 \pm 17,3$ & $231,1 \pm 16,6$ & $210,8 \pm 21,8$ & $231,4 \pm 31,4$ \\
\hline $\begin{array}{l}\text { Aceite linaza } \\
(n=8)^{*}\end{array}$ & $97,6 \pm 7,6$ & $129,6 \pm 5,0$ & $153,6 \pm 5,9$ & $184,9 \pm 12,7$ & $196,8 \pm 12,9$ & $209,3 \pm 10,2$ & $232,4 \pm 14,5$ & $212,0 \pm 20,2$ & $234,9 \pm 26,3$ \\
\hline$p$ & ns & ns & ns & ns & ns & ns & ns & ns & ns \\
\hline
\end{tabular}

ns: diferencia no significativa. ${ }^{*}$ media \pm desviación estándar. 
Respecto a los parámetros séricos se observa que a los 30 días de seguimiento, existen diferencias significativas entre los grupos respecto a colesterol total, HDL y triglicéridos. En el caso de las mediciones a los 60 días, no se observó diferencias estadísticamente significativas entre los grupos, en ninguno de los parámetros; no obstante, se observó valores en promedio más bajos en colesterol total en el grupo SI05 con respecto al grupo control SSF y L05; en cuanto a HDL, los valores más altos se presentaron en el grupo SI05, mientras que los valores más bajos de triglicéridos se observaron en el grupo L05, con respecto a los valores de glucosa, urea y TGP los valores más bajos se encontraron en el grupo SI05, y en fosfatasa alcalina el grupo SI05 presentó los valores más altos (Tabla 4).

\section{DL5O DE LOS ACEITES CRUDOS}

Tras la administración de los aceites crudos de sacha inchi y linaza para determinar la DL50, se observó los ratones que recibieron aceite de sacha inchi hasta 1 $\mathrm{mL} / \mathrm{kg} /$ día no mostraron signos de toxicidad alguna, mientras que los ratones que recibieron aceite de linaza en la dosis de $1 \mathrm{~mL} / \mathrm{kg} /$ día mostraron sudoración profusa pero, luego de dos horas se estabilizaron, permaneciendo así todos los animales hasta los 14 días de observación. Si la densidad del aceite de sacha inchi fue de $0,9269 \mathrm{~g} / \mathrm{mL}$ y el peso del ratón al concluir el experimento fue de $25 \mathrm{~g}$, se demuestra que la DL50 se encuentra sobre los $37076 \mathrm{mg} / \mathrm{kg}$ de masa corporal; para el caso del aceite de linaza la DL50 se encuentra sobre los $37240 \mathrm{mg} / \mathrm{kg}$ debido a que su densidad fue de $0,931 \mathrm{mg} / \mathrm{mL}$.

\section{DISCUSIÓN}

En cuanto a las características químicas de los aceites, encontramos un índice de refracción y densidad del aceite crudo de sacha inchi dentro de los valores reportados por Ángeles ${ }^{(19)}$, Mejía ${ }^{(20)}$ y la Asociación de Productores Industriales para la comercialización de productos del sacha inchi (APISI) ${ }^{21)}$; mientras que los valores de ácidos grasos libres fueron similares a los reportados por la (APISI) ${ }^{(21)}$ e inferiores a los encontrados por Ángeles ${ }^{(19)}$ y Mejía ${ }^{(20)}$, lo que indica acidez baja y calidad óptima para ser comercializados, el mismo análisis es válido para el aceite crudo de linaza.

Teniendo en cuenta la composición de los ácidos grasos en los aceites de sacha inchi y linaza, los valores del índice de yodo deben ser relativamente altos; contradictoriamente APISI (21), dentro del documento del Comité de Normas Técnicas del sacha inchi, reporta valores relativamente bajos de alrededor de $140 \mathrm{~g}$ de I/100g; por otro lado Ángeles (19) y Mejía (20) reportan valores de 174,8 y $189,0 \mathrm{~g}$ de $\mathrm{I} / 100 \mathrm{~g}$ de aceite crudo respectivamente, valores similares a los encontrados en esta investigación que reporta para el aceite de sacha inchi y linaza valores de 194,0 y 188,7 g de I/100g y que están de acuerdo con los valores reportados por la literatura para el caso de la linaza ${ }^{(4)}$.

En cuanto a los valores de índice de saponificación, los encontrados en este estudio para el aceite de sacha inchi son ligeramente inferiores a los hallados por APISI(21), quienes reportan valores entre 192,5 y $195,9 \mathrm{mg} \mathrm{KOH} / \mathrm{g}$, para el caso del aceite de linaza este se encuentra dentro del rango reportado por Cuppett ${ }^{(4)}$; asimismo el valor

Tabla 4. Parámetros bioquímicos séricos de los animales a los 30 y 60 días.

\begin{tabular}{|c|c|c|c|c|c|c|c|}
\hline Grupos & $\begin{array}{c}\mathrm{CT} \\
(\mathrm{mg} / \mathrm{dL})\end{array}$ & $\begin{array}{c}\mathrm{HDL} \\
(\mathrm{mg} / \mathrm{dL})\end{array}$ & $\begin{array}{c}\text { TG } \\
(\mathrm{mg} / \mathrm{dL})\end{array}$ & $\begin{array}{c}\mathbf{G} \\
(\mathrm{mg} / \mathrm{dL})\end{array}$ & $\begin{array}{c}U \\
(\mathrm{mg} / \mathrm{dL})\end{array}$ & $\begin{array}{c}\text { TGP } \\
\text { (Ul/dL) }\end{array}$ & $\begin{array}{c}\text { FA } \\
\text { (Ul/dL) }\end{array}$ \\
\hline \multicolumn{8}{|c|}{ Evaluación a los 30 días } \\
\hline $\operatorname{SSF}(n=8)^{*}$ & $155,3 \pm 26,2 \mathrm{ab}$ & $48,3 \pm 4,7^{b}$ & $124,3 \pm 23,3^{b}$ & $83,5 \pm 12,2$ & $17,3 \pm 2,2$ & $16,0 \pm 3,6$ & $131,4 \pm 30,1$ \\
\hline $\operatorname{SI05}(n=8)^{*}$ & $180,3 \pm 18,2^{a}$ & $53,5 \pm 9,0$ ab & $151,3 \pm 22,8^{a}$ & $83,5 \pm 4,3$ & $18,0 \pm 5,4$ & $14,8 \pm 3,5$ & $132,0 \pm 33,6$ \\
\hline L05 $(n=8)^{*}$ & $142,3 \pm 27,8^{b}$ & $56,5 \pm 3,4^{a}$ & $130,5 \pm 17,4$ ab & $87,8 \pm 8,7$ & $15,5 \pm 3,3$ & $15,5 \pm 2,9$ & $132,5 \pm 24,9$ \\
\hline$p$ & $<0,01$ & $<0,05$ & $<0,05$ & ns & ns & ns & ns \\
\hline \multicolumn{8}{|c|}{ Evaluación a los 60 días } \\
\hline $\operatorname{SSF}(n=8)^{*}$ & $146,5 \pm 23,5$ & $44,5 \pm 5,1$ & $155,6 \pm 38,2$ & $88,0 \pm 8,4$ & $18,4 \pm 3,7$ & $19,0 \pm 12,0$ & $139,6 \pm 31,9$ \\
\hline $\operatorname{SIO5}(n=8)^{*}$ & $138,0 \pm 20,4$ & $49,5 \pm 8,5$ & $146,8 \pm 29,8$ & $81,0 \pm 9,5$ & $15,4 \pm 3,9$ & $16,9 \pm 8,3$ & $142,8 \pm 45,5$ \\
\hline $\operatorname{LO} 5(n=8)^{*}$ & $146,9 \pm 10,6$ & $47,9 \pm 5,8$ & $139,1 \pm 7,0$ & $81,8 \pm 5,8$ & $18,1 \pm 8,3$ & $16,1 \pm 7,8$ & $136,4 \pm 40,0$ \\
\hline$p$ & ns & ns & ns & ns & ns & ns & ns \\
\hline
\end{tabular}

SSF: solución salina de suero fisiológico; SI05: aceite de sacha inchi 0,5 mL; L05: aceite de linaza 0,5 mL; CT: colesterol total; HDL: lipoproteínas de alta densidad; TG: triglicéridos; G: glucosa; U: úrea; TGP: alanino amino transferasa; FA: fosfatasa alcalina. p: valor $\mathrm{p}$ del análisis de varianza; ns: diferencia no significativa. * media \pm desviación estándar. Valores con diferentes letras dentro de cada columna denotan diferencias significativas en la prueba de Tukey con $p \leq 0,05$. 


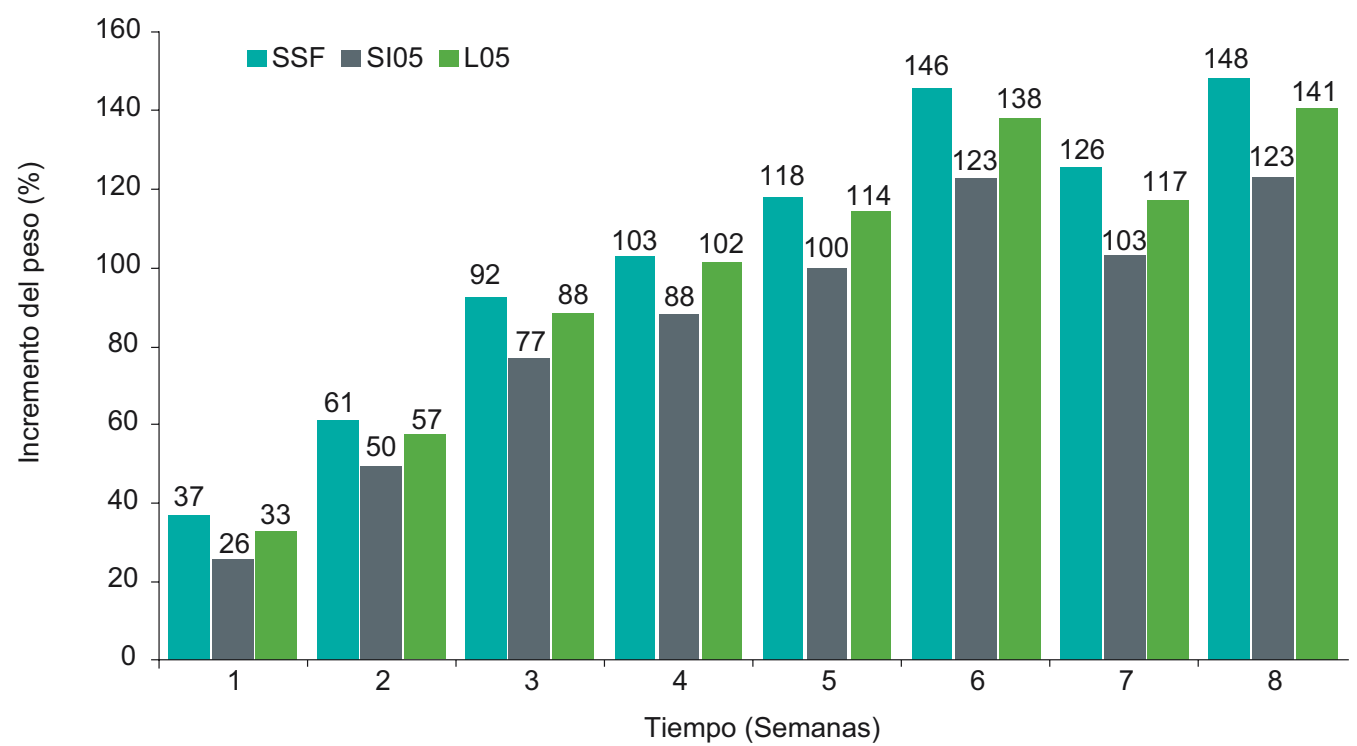

Figura 2. Incremento del peso corporal de los animales en los diferentes grupos experimentales. SSF: solución salina de suero fisiológico, SI05:aceite de sacha inchi 0,5 mL, L05:aceite de linaza 0,5 mL. Cada barra representa los valores promedio de incremento de peso de ocho animales.

de peróxido encontrado en la investigación presenta promedios inferiores a los reportados por APISI en el documento de los requisitos para el aceite de sacha inchi, con excepción del valor que reportan para Tres Unidos, cabe recalcar que los valores reportados por APISI en el documento indicado, no precisan datos de altitud, latitud ni longitud, así como valores promedio ${ }^{(21)}$.

El análisis del contenido de ácidos grasos se realizó con la metodología implementada en el Laboratorio del Instituto Tecnológico Pesquero del Perú (ITP). En la Tabla 2, se presenta los resultados de nuestro estudio y se compara con los obtenidos por Hamaker ${ }^{(15)}$ y Follegati-Romero(16) en el caso de sacha inchi, y lo reportado por Rallidis ${ }^{(17)}$ y Clandinin ${ }^{(18)}$ en el caso del aceite de linaza. Podemos notar que los componentes principales -ácido oleico, linoleico y linolénico- en el sacha inchi, son valores dentro de los rangos descritos en esas publicaciones. Puede observarse, que la linaza presenta valores superiores de ácido oleico y linolénico, contrario a lo que ocurre con el ácido linoléico donde son inferiores (Tabla 2).

Al evaluar la toxicidad oral de los aceites crudos, se midió los pesos corporales de los animales como síntoma de toxicidad, para cada una de las ocho semanas del experimento, no existen diferencias significativas entre los tres grupos experimentales (SSF, SI05 y L05), ello se traduce en que los pesos corporales se encontraron dentro de los valores establecidos para la curva de crecimiento de la especie y línea en estudio (Figura 1). No obstante, cuando se calculó el incremento de acuerdo con la expresión indicada en la sección de métodos, el grupo al que se suministró aceite de sacha inchi (SI05) presentó el menor incremento de peso durante las ocho semanas de seguimiento (Figura 2).

Al respecto Burdge y Wootton, en las investigaciones sobre la conversión de ácido a-linolénico a ácido palmítico, palmitoléico, esteárico y oleico en hombres y mujeres, así como la conversión hacia ácido eicosapentaenoico, docosapentaenoico y docosahexaenoico en mujeres jóvenes, indican tres procesos principales entre los que se encuentran la desaturación de los dobles enlaces acompañada de elongaciones subsecuentes hacia ácidos grasos de cadena más larga y la oxidación- $\beta$ del omega-3 hacia la conversión de Acetil CoA y desde aquí vía procesos oxidativos hacia la conversión de $\mathrm{CO}_{2}$ o síntesis de otros ácidos de cadena corta como C16:0, C16:1n-7, C18:0 y C18:1n-9 que se acumulan dentro del organismo. Se considera que en este estudio se observó una conversión eficiente de los ácidos grasos del aceite de sacha inchi hacia $\mathrm{CO}_{2}$ vía procesos de oxidación- $\beta$ debido al menor incremento del peso corporal con respecto al grupo control (SSF) y de aceite de linaza (L05) ${ }^{(22) .}$

En relación con los contenidos de colesterol total y lipoproteínas a nivel sérico, en los grupos experimentales a los 30 y 60 días, aquellos que recibieron aceite de sacha inchi $0,5 \mathrm{~mL} / \mathrm{kg}$ animal/día presentaron los menores valores promedio de colesterol total hacia los 60 días (138,0 $\mathrm{mg} / \mathrm{dL}$ de colesterol total), mientras que los triglicéridos fueron menores en el grupo al que se administró aceite de linaza $0,5 \mathrm{~mL} / \mathrm{kg}$ de animal/día a los 60 días $(139,1$ $\mathrm{mg} / \mathrm{dL}$ de triglicéridos). Con respecto a los valores promedio de lipoproteínas de alta densidad, estos fueron mayo- 

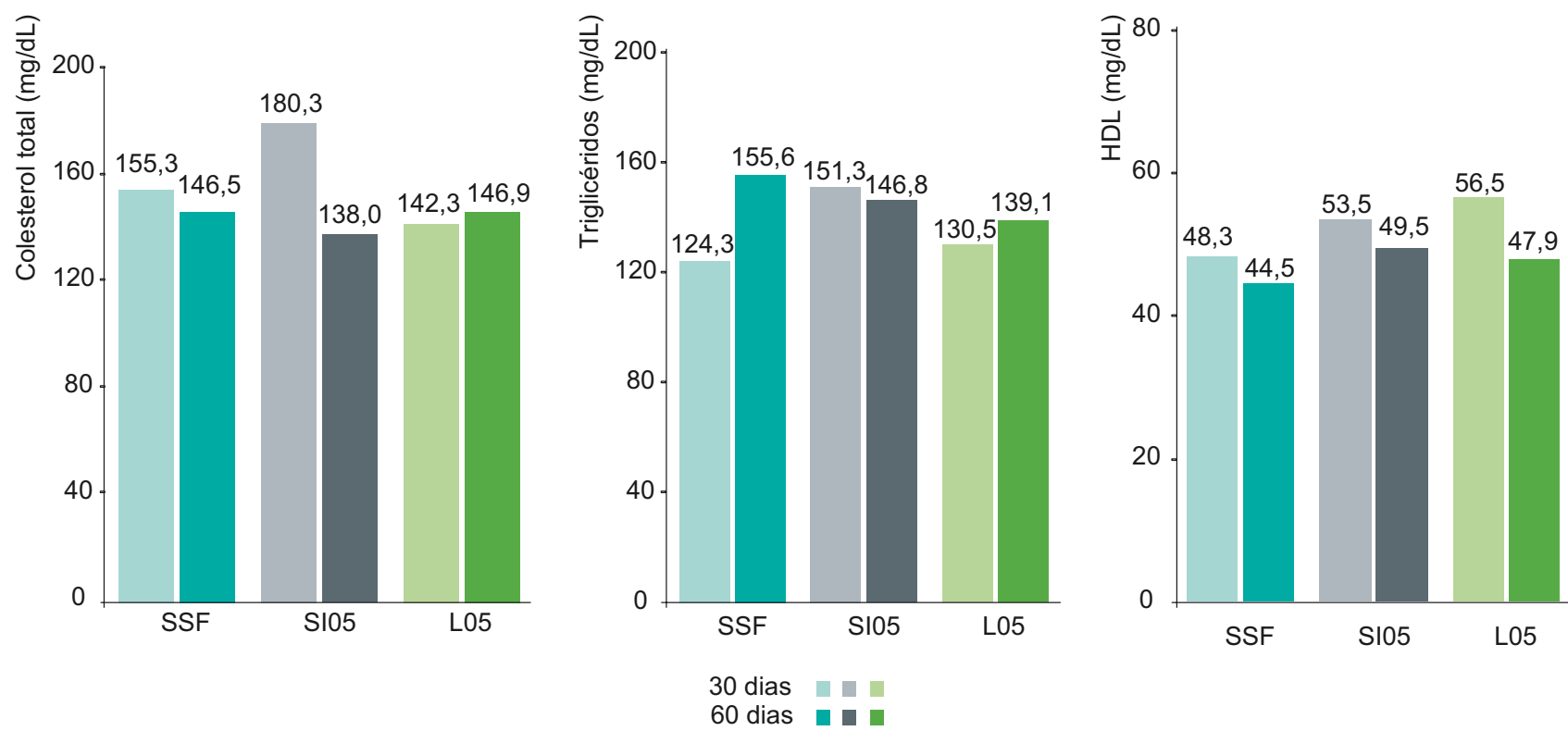

Figura 3. Niveles de colesterol, triglicéridos y HDL en $\mathrm{mg} / \mathrm{dL}$ de los grupos experimentales. SSF: solución salina de suero fisiológico, SI05: aceite de sacha inchi 0,5 mL, L05: aceite de linaza 0,5 mL. Cada barra representa los valores promedio de incremento de peso en ocho animales.

res a los 60 días de consumo diario en el grupo al que se administró aceite de sacha inchi $0,5 \mathrm{~mL} / \mathrm{kg}$ de animal/ día (49,5 mg/dL de HDL) (Figura 3).

De acuerdo con estos resultados, se demuestra, por un lado, que los aceites de sacha inchi y linaza no presentan efectos tóxicos al ser consumidos y, por el otro, se ve un efecto benéfico en la disminución, a los 60 días de consumo, de colesterol total y triglicéridos, mientras que el marcador HDL se incrementó durante el estudio; lo que podría vislumbrar un potencial efecto en la prevención de eventos cardiovasculares $(17,18,22,23)$.

En ese sentido, aunque se trata de un modelo animal, el consumo de los ácidos grasos poliinsaturados de los aceites de sacha inchi y linaza, coinciden con lo reportado por otros investigadores ${ }^{(24,25)}$. En humanos, se desarrolló una investigación clínica acerca del consumo de ácidos grasos omega-3 en relación a la composición de ésteres de colesterol LDL y colesterol LDL, donde se encontró valores promedio superiores de triglicéridos para el aceite de oliva y linaza con relación al aceite de pescado y valores promedio similares para colesterol total, colesterol LDL y colesterol HDL al finalizar el estudio (Tabla 5) ${ }^{(25)}$.

Los otros parámetros bioquímicos como glucosa, úrea, TGP y fosfatasa alcalina a los 30 y 60 días según el análisis de varianza y prueba de significancia de Tukey con $p \leq 0,05$ no mostraron diferencias significativas en los grupos del control (SSF), aceite de sacha inchi (SI05) y linaza (L05); sin embargo, debemos indicar que los valores de glucosa a los 30 y 60 días en el grupo de animales a los que se administró aceite de sacha inchi $0,5 \mathrm{~mL} / \mathrm{kg}$ de animal/día fueron menores con respecto al grupo control y de aceite de linaza en la misma dosis,

Tabla 5. Niveles de triglicéridos y lipoproteínas con colesterol en plasma sanguíneo.

\begin{tabular}{lcccc}
\hline Fracción & Aceite de oliva & Aceite de pescado & Aceite de linaza & p \\
\hline $\mathrm{TG}(\mathrm{mmol} / \mathrm{L})(\mathrm{n}=26)^{*}$ & $0,94 \pm 0,06^{\mathrm{a}}$ & $0,70 \pm 0,03^{\mathrm{b}}$ & $0,97 \pm 0,08^{\mathrm{a}}$ & $<0,05$ \\
$\mathrm{TC}(\mathrm{mmol} / \mathrm{L})(\mathrm{n}=26)^{*}$ & $4,56 \pm 0,17$ & $4,61 \pm 0,18$ & $4,59 \pm 0,15$ & $\mathrm{~ns}$ \\
$\mathrm{LDL}_{\mathrm{c}}(\mathrm{mmol} / \mathrm{L})(\mathrm{n}=26)^{*}$ & $2,57 \pm 0,15$ & $2,73 \pm 0,15$ & $2,64 \pm 0,13$ & $\mathrm{~ns}$ \\
$\mathrm{HDL}_{\mathrm{c}}(\mathrm{mmol} / \mathrm{L})(\mathrm{n}=26)^{*}$ & $1,54 \pm 0,06$ & $1,55 \pm 0,07$ & $1,50 \pm 0,06$ & $\mathrm{~ns}$ \\
\hline
\end{tabular}

Adaptado de: Wang et al.

TG: triglicéridos, TC: colesterol total, LDLC: lipoproteínas de baja densidad con colesterol, HDLC: lipoproteínas de alta densidad con colesterol total.

p: valor de significancia para análisis de varianza. Valores con diferentes letras dentro de cada fila denotan diferencias significativas en la prueba de Duncan con $p \leq 0,05$.

${ }^{*}$ media \pm desviación estándar. 
resultados que se encuentra de acuerdo con el estudio realizado por Zhang et al. ${ }^{(26)}$ donde los investigadores concluyeron que el ácido $\alpha$-linolénico ejerce un efecto antiapoptótico y de este modo ser considerado como un agente terapéutico potencial para el tratamiento de complicaciones de diabetes cardiovascular.

Cuando se evaluó la dosis letal mediana (DL50), la administración de sacha inchi y linaza, por por vía oral por mas de $37 \mathrm{~g} / \mathrm{kg}$ del peso corporal, no produjo signos de toxicidad o muerte en los ratones, sugiriendo una DL50 sobre los $37 \mathrm{~g} / \mathrm{kg}$, lo que está de acuerdo con lo descrito por Kennedy et al. (27) y Costa-Silva et al. (28), es decir, las sustancias que presentan una DL50 por encima de los $5 \mathrm{~g} / \mathrm{kg}$ por vía oral son consideradas prácticamente no tóxicas. Además, de acuerdo con las normas de la Comunidad Europea que en la clasificación de la toxicidad aguda oral, cuando se supera la dosis de $2000 \mathrm{mg} / \mathrm{kg}$ se considera como 'no clasificado'(no tóxico) ${ }^{(29)}$

Se concluye que los aceites de sacha inchi y linaza son inocuos a los sesenta días de consumo y tienen una DL50 superior a $37 \mathrm{~g} / \mathrm{kg}$. No obstante, este estudio presenta algunas limitaciones relacionadas con el tamaño muestral que no fue calculado previo a su ejecución. Se sugiere realizar estudios que puedan incluir un mayor número de individuos de investigación, asimismo, iniciar estudios observacionales en personas para cuantificar el real consumo en la población, previo a la evaluación experimental en seres humanos.

\section{AGRADECIMIENTOS}

A Consejo Nacional de Ciencia y Tecnología (CONCYTEC), Deutsche Gesellschaft für Technische Zusammenarbeit (GTZ) y Fundación para el Desarrollo Agrario (FDA).

\section{Fuente de Financiamiento}

Programa de desarrollo rural sostenible y apoyo técnico de la cooperación alemana (PDRS-GTZ). Secretaria de Economía Cooperación Suiza (SECO) en apoyo al Proyecto Nacional PeruBiodiverso.

\section{Conflictos de Interés}

Los autores declaran no tener conflictos de interés en la ejecución y publicación de este artículo.

\section{REFERENCIAS BIBLIOGRÁFICAS}

1. Brack A. Diccionario enciclopédico de plantas útiles de Perú. Cusco: Centro Bartolomé de las Casas; 1999.
2. Arévalo G. Colección, caracterización y mantenimiento de germoplasma de oleaginosas nativas. Estación Experimental Agraria El Porvenir - Instituto Nacional de Innovación Agraria (INIA). Informes Anuales 1990-1995. Tarapoto: INIA; 1996.

3. Guillén M, Ruiz A, Cabo N, Chirinos R, Pascual G. Characterization of sacha inchi (Plukenetia volubilis L.) oil by FTIR spectroscopy and 1H NMR. Comparison with linseed oil. Comparison with linseed oil. JAOCS. 2003;80(8):755762.

4. Cuppett S. Oil quality indices. In: Wrolstad RE, Acree TE, An H, Decker EA, Penner MH, Reid DS, et al. (eds). Current protocols in food analytical chemistry. New York: John Wiley \& Sons, Inc.; 2001. p. D1.4.1- D1.4.3

5. El-Rahim $\mathbf{A H}$, Hafiz NA. Investigation on the protective effect of Grape seed and Linseed oils against ciclophosphamide induced genotoxicity in mice. Global Veterinaria. 2009;3(5):377-82.

6. Barceló-Coblijn G, Murphy EJ. Alpha-linolenic acid and its conversion to longer chain n-3 fatty acids: benefits for human health and a role in maintaining tissue n-3 fatty acid levels. Prog Lipid Res. 2009;48(6):355-74.

7. Ihara-Watanabe M, Umekawa H, Takahashi T, Furuichi Y. Effects of dietary alpha- or gamma-linolenic acid on levels and fatty acid compositions of serum and hepatic lipids, and activity and mRNA abundance of 3-hydroxy-3-methylglutaryl CoA reductase in rats. Comp Biochem Physiol A Mol Integr Physiol. 1999;122(2):213-20.

8. Association of Official Analytical Chemists (AOAC). AOAC official methods of analysis. Arlington, VA: AOAC; 2005

9. Instituto Tecnológico Pesquero del Perú (ITP), Laboratorio de Análisis Fisicoquímico. Composición de ácidos grasos por cromatografía de gases. Lima: ITP; 2003.

10. Vega R, Carrillo C. Efecto sobre la motilidad intestinal y toxicidad aguda oral del extracto fluido de Ocimun gratissimum L. (orégano cimarrón). Rev Cubana Plant Med. 1997;2(2-3):14-8.

11. Institute for Laboratory Animal Research. Guide for the care and use of laboratory animals. 8th Ed. Washington, DC: National Academy Press; 2010.

12. Lima LB, Vasconcelos CF, Maranhão HM, Leite VR, Ferreira PA, Andrade BA, et al. Acute and subacute toxicity of Schinus terebinthifolius bark extract. J Ethnopharmacol. 2009;126(3):468-73

13. Organization for Economic Co-operation and Development (OECD). Guideline for the testing of chemicals. Repeated dose 28-day oral toxicity study in rodents 407. Paris: OECD; 1995.

14. Biswas TK, Sana NK, Badal RK, Huque EM. Biochemical study of some oils seeds (Brassica, sesame and linseed). Pakistan Journal of Biological Science. 2001;4(8):1002-5.

15. Hamaker BR, Valles C, Gilman R, Hardmeier RM, Clark D, García HH, et al. Amino Acid and Fatty Acid Profiles of the Inca Peanut (Plukenetia volubilis). Cereal Chem. 1992;69(4):461-3.

16. Follegatti-Romero LA, Piantino CR, Grimaldi R, Cabral FA. Supercritical CO2 extraction of omega-3 rich oil from Sacha inchi (Plukenetia volubilis L.) seeds. J Supercrit Fluid. 2009;49(3):323-9 
17. Rallidis LS, Paschos G, Liakos GK, Velissaridou AH, Anastasiadis G, Zampelas A. Dietary alpha-linolenic acid decreases $C$-reactive protein, serum amyloid $A$ and interleukin-6 in dyslipidaemic patients. Atherosclerosis. 2003;167(2):237-42.

18. Clandinin MT, Foxwell A, Goh YK, Layne K, Jumpsen JA. Omega-3 fatty acid intake results in a relationship between the fatty acid composition of LDL cholesterol ester and LDL cholesterol content in humans. Biochim Biophys Acta. 1997;1346(3):247-52.

19. Ángeles M. Determinación de la estabilidad del aceite crudo y semi refinado de la semilla de sacha inchi (Plukenetia volubilis L.) sometido a temperaturas variables de almacenamiento. [Tesis para optar el Título de Ingeniero de Industrias Alimentarias]. Lima: Facultad de Industrias Alimentarias; Universidad Nacional Agraria La Molina; 2002.

20. Mejía M. Extracción y refinación del aceite de sacha inchi. [Tesis para optar el Título de Ingeniero de Industrias Alimentarias]. Lima: Facultad de Industrias Alimentarías, Universidad Nacional Agraria La Molina; 1997.

21. Asociación de Productores Industriales para la comercialización de productos del sacha inchi (APISI). Norma técnica del sacha inchi. Lima: APISI; 2010.

22. Burdge GC, Wootton SA. Conversion of $\alpha$-linolenic acid to palmitic, palmitoleic, stearic and oleic acid in men and women. Prostaglandins Leukot Essent Fatty Acids. 2003;69(4):283-90.

23. Brewer HB. Increasing HDL cholesterol levels. N Engl J Med. 2004;350(15):1491-4.

24. Carrero JJ, Martín-Bautista E, Boró L, Fonollá J, Jiménez J, Boza JJ, et al. Efectos cardiovasculares de los ácidos grasos omega-3 y alternativas para incrementar su ingesta. Nutr Hosp. 2005;20(1):63-9.
25. Wang Ch, Harris WS, Chung M, Lichtesntein AH, Balk EM, Kupelnick B, et al. n-3 Fatty acids from fish or fish-oil supplements, but not $\alpha$-linolenic acid, benefit cardiovascular disease outcomes in primary-and secondary-prevention studies: a systematic review. Am J Clin Nutr. 2006;84(1):5-17.

26. Zhang W, Wang $R$, Han SF, Bu L, Wang SW, Ma $H_{\text {, }}$ et al. Alpha-linolenic acid attenuates high glucoseinduced apoptosis in cultured human umbilical vein endothelial cells via PI3K/Akt/eNOS pathway. Nutrition. 2007;23(10):762-70.

27. Kennedy GL, Ferenz RL, Burgess BA. Estimation of acute oral toxicity in rates by determination of the approximate lethal dose rather than the LD50. J Appl Toxicol. 1986;6(3):145-8.

28. Costa-Silva JH, Lima CR, Silva EJ, Araújo AV, Fraga MC, Ribeiro E Ribeiro A, et al. Acute and subacute toxicity of the Carapa guianensis Aublet (Meliaceae) seed oil. J Ethnopharmacol.. 2008;116(3):495-500.

29. Unión Europea. Decisión 2000/368/CE de la Comisión: aproximación de las disposiciones legales, reglamentarias y administrativas en materia de clasificación, embalaje y etiquetado de las sustancias peligrosas. Diario Oficial. 2000;L136:108-25.

Correspondencia: Dra. Arilmi Gorriti Gutierrez. Correo electrónico: arilmigorritig@gmail.com

\section{Consulte las ediciones anteriores de la}

\section{Revista Peruana de Medicina Experimental y Salud Pública en}

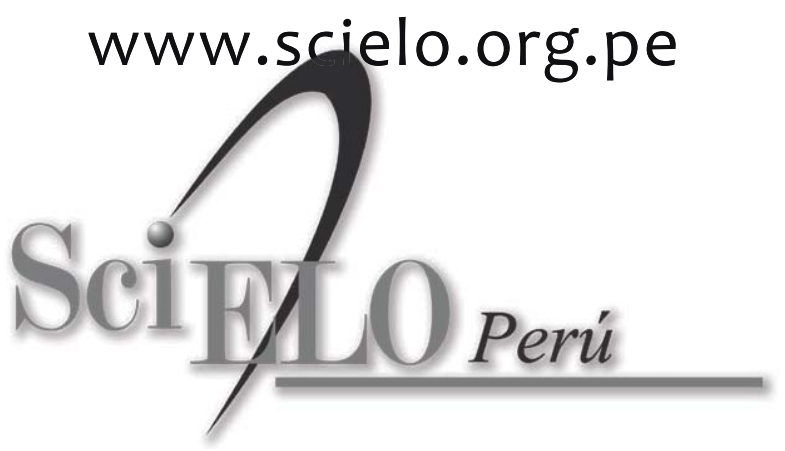

